\title{
HOW MODIFICATIONS IN GOALS IN SMALL-SIDED AND CONDITIONED GAMES IN SOCCER INFLUENCE THE TACTICAL ACTIONS OF YOUNG SOCCER PLAYERS
}

original paper

( ) University School of Physical Education in Wroclaw

DOI: https://doi.org/10.5114/hm.2021.100328

\section{LUCAS OMETTO $^{1}$, ISRAEL TEOLDO ${ }^{2}$, DAVI SILVA ${ }^{2}$, FABRÍCIO VASCONCELLOS ${ }^{1}$}

${ }^{1}$ Post-Graduate Program in Exercise and Sport Sciences, Laboratory of Soccer Studies, University of Rio de Janeiro State, Rio de Janeiro, Brazil

${ }^{2}$ Centre of Research and Studies in Soccer, Universidade Federal de Viçosa, Viçosa, Brazil

\begin{abstract}
Purpose. This study analysed the influence of the absence of goalkeeper and the number of goals in tactical actions in 3 configurations of small-sided and conditioned games (SSCGs): (a) goalkeeper + 3 vs. 3 + goalkeeper; (b) 3 vs. 3 (no goalkeeper); and (c) 3 vs. 3 with 2 mini goals.

Methods. Overall, 24 under-15 soccer players were divided into 8 teams of 3 players each. Seven games were played for each configuration for a total of 21. The tactical behaviour was evaluated with the System of Tactical Assessment in Soccer (FUT-SAT).

Results. More offensive actions were performed in the SSCGs with goalkeepers. The games played without goalkeepers led to more actions associated with defensive unity but also a higher rate of errors concerning the tactical principles of delay and defensive unity. In relation to the outcome of actions, more fouls, throw-ins, and corner kicks were observed in the SSCGs with 2 mini goals.

Conclusions. The games played without goalkeepers reflected a higher difficulty in managing spaces of the game and led to a higher number of errors in the performance of core defensive tactical principles. In the games played with 2 mini goals, it was observed that the players on the wings were positioned closer to each other, which made the play more fragmented compared with the other conditions. In accordance with these results, it is possible to identify the influence of goalkeepers on tactical behaviour during SSCGs.
\end{abstract}

Key words: small-sided and conditioned games, tactical behaviour, constraints, task constraints, soccer

\section{Introduction}

Small-sided and conditioned games (SSCGs) are used by coaches because of the possibility to modify the formal and functional structure of the game of soccer $[1,2]$. Generally, these modifications occur through the manipulation of task constraints (e.g. number of players, goals, rules) in the game that condition the physical [3], technical [4], and tactical actions performed by soccer players [5, 6]. More specifically, tactical actions can simply be understood as the management of space through positioning and movements performed by the players and teams [7].

The manipulation of the task constraints can pro- voke the emergence of individual $[8,9]$ and collective behaviour patterns [10]. Accordingly, SSCGs offer different possibilities to change the objective of training exercise by manipulating task constraints $[11,12]$. Amongst these constraints, modifying targets has been a widely utilized solution for coaches to develop inherent aspects to the game [3]. As presented in the next paragraphs, it has been shown that changing goals can change tactical actions, where goals can be seen as the nets where players should strike to score points or as the objectives to pursue in the play $[2,13,14]$. In the present study, the analysis was directed towards changes in the position and use of the goals as nets where to strike in for the aforementioned arguments.

Correspondence address: Fabrício V.A. Vasconcellos, Institute of Physical Education and Sports, Laboratory of Physical Activity and Health Promotion, University of Rio de Janeiro State, Rua São Francisco Xavier 524 / sala 8121F - Maracanã, Rio de Janeiro, RJ, Brazil, CEP: 20550-013, e-mail: fabriciovav@hotmail.com

Received: April 24, 2020

Accepted for publication: August 10, 2020

Citation: Ometto L, Teoldo I, Silva D, Vasconcellos F. How modifications in goals in small-sided and conditioned games in soccer influence the tactical actions of young soccer players. Hum Mov. 2021;22(3):92-100; doi: https://doi.org/10.5114/ hm.2021.100328. 
According to Teoldo et al. [14], in games with small goals, there were more movements performed by the carrier of the ball in the direction to the opponent goal, more movements to utilize and amplify the effective space of play, and more movements that allowed the team to attack as a unity in offensive phases. In the defensive phases, there were more movements of direct opposition to the ball carrier and movements that increased the goal protection and facilitated the recovery of the ball possession. This results in games with small goals to increase the tactical principles that have a direct relation with the ball and to lead the amplification of the effective space of play.

In addition, the study conducted by Travassos et al. [2] evaluated how changes in the number and size of the goals influenced the spatial-temporal relations in players in each corridor (left, right, and central) and sector (central and defensive) in SSCGs. The results highlighted a greater distance between the teams' centroids in corridors in SSCGs played with more goals (e.g. 2 goals to protect for each team). Moreover, Castellano et al. [15] sought to identify how changes in the number of goals and floaters on the sideline influenced the size of the area that was covered by the team in both offensive and defensive phases. It was observed that the size of the area covered by the teams in the offensive phase was greater than in the defensive phase in a 7 vs. 7 game with a goalkeeper and 2 floaters, whereas the size of the covered area was similar in both phases in the game played with 2 mini goals. Also, Ometto et al. [16] compared the time of ball possession in each corridor in different configurations of the game (e.g. with goalkeepers, without goalkeepers, with 2 mini goals) and found that players explored the corridors for more time in order to score points in the SSCGs. In brief, these results confirm the fact that changing goals influences the possibilities of actions in SSCGs.

Despite the contribution of the studies in the understanding of tactical actions in SSCGs, the results are still incipient since only one study has enlightened how the actual modifications in the goals influenced the tactical actions based on core tactical principles of the game [17]. Additionally, few studies intended to specifically investigate the influence of changing the position of goals on players' tactical behaviour [18, 19]. It is still relevant to highlight that a study based on this framework is important to understand how the SSCGs can offer players the possibility to reach better tactical solutions to problems upcoming in the game, thus improving the understanding of the game [13].

As far as the literature is concerned, no study on SSCGs has considered the goalkeeper in the analysis of the tactical actions. Thus, there is still no objective information on the influence of the presence and absence of goalkeepers on the tactical actions performed by the teammates and opponents in both offensive and defensive phases of game. It is relevant to note that the manipulation of goals and goalkeeper absence can make the tactical actions easier or more difficult and, in addition, change the information that players can use to explore the possibilities of action in the play [9]. Because of the lack of evidence in this matter, the present study analysed the influence of the absence of the goalkeeper and the number of goals in tactical actions in 3 configurations of SSCGs: (a) goalkeeper + 3 vs. $3+$ goalkeeper; (b) 3 vs. 3 (no goalkeeper); and (c) 3 vs. 3 with 2 mini goals.

\section{Material and methods}

\section{Sample}

The sample was composed of 24 players of the under-15 age level $\left(M_{\text {age }}=14.67\right.$ years, $\left.S D=0.30\right)$ affiliated to a first division club of the Brazilian Championship of 2016; it was a non-probabilistic sample selection. The players performed 9570 offensive and defensive tactical actions in 21 SSCGs. On average, the players were $169.78 \pm 7.6 \mathrm{~cm}$ high, weighted $60.91 \pm$ $7.4 \mathrm{~kg}$, had $7.31 \pm 3.0$ years of practice in soccer and had been affiliated to the club for $21.60 \pm 10.6$ months.

As an inclusion criterion, the players should have had minimally 6 months of systematic training at the club, subscribing to a program offering training sessions with an average duration of 90 minutes. The players who were injured or were back from an injury were excluded. All legal tutors responsible of the players were informed of the objectives and possible risks of the study.

\section{Data collection instrument}

The instrument used to assess the tactical actions was the System of Tactical Assessment in Soccer (FUT-SAT), validated by Teoldo et al. [17]. This system consists of a field test (goalkeeper +3 vs. 3 + goalkeeper) with proportionally reduced pitch dimensions $(36 \times 27 \mathrm{~m})$ played for 4 minutes. The reason for choosing these game configurations was the premise that the goalkeeper +3 vs. $3+$ goalkeeper respects the minimal configuration that guarantees the occurrence of all tactical principles of game [20]. However, the configuration of the test can be changed without the system losing its essence, which is based on the 10 core 
tactical principles of the game of soccer [7]. These principles are divided between the offensive phase: (i) penetration, progression movements of the ball carrier toward the goal and/or the opposing bottom line; (ii) offensive coverage, movements of support to the ball carrier; (iii) depth mobility, movements of players between the last defender and goal line; (iv) width and length, movements for use and expansion of the effective game space; and (v) offensive unity, movements that allow the teams to attack in unity; and the defensive phase: (i) delay, movements of direct opposition to the ball carrier; (ii) defensive coverage, movements of support of the player who directly opposes the ball carrier; (iii) balance, movements that ensure defensive stability in the area of the ball dispute; (iv) concentration, movements that increase the goal protection and facilitate the recovery of the ball possession; and (v) defensive unity, movements that allow the team to defend in unity [7]. All the tactical actions executed by the players were filmed for further analysis with the Soccer Analyser ${ }^{\circledast}$ software.

\section{Material}

The games were recorded with a Sony digital camera, model Sony HDR CX 220, positioned in the diagonal between the sideline and the goal line. The images were transferred to a Lenovo computer for processing and were analysed with the use of the Soccer Analyser ${ }^{\circledR}$ software. This software allows the insertion of dynamic and static spatial references that enable the analysis of the tactical behaviour of soccer players based on the implementation of the core tactical principles of the soccer game.

\section{Data collection procedure}

The players were divided into 8 teams of 3 players each. Three goalkeepers played in rotation in the games that involved their position. However, they were not analysed by the system since their actions differ from the ones performed by the field players. The composition of the teams was determined by the coaching staff with the aim to balance them. To increase players' motivation and, consequently, their involvement in the game, the games were organized in a play-off structure (round of 8 , semis, final), totalizing 7 games per configuration. Three visits were held to conduct data collection: the games with goalkeepers were played on the first visit; the games without goalkeepers on the second; and the games with 2 mini goals on the third. All tournaments constituted the only activity of the session when the visit occurred, which helped avoid fatigue on the day after. The tournament began after a usual 15-minute warm-up exercise as normally operated by the club. All games were played for 4 minutes, in a $36 \times 27 \mathrm{~m}$ field with natural grass surface, respecting the official rules of the game of soccer except the off-side rule.

The players wore coloured vests with numbers from 1 to 6 . All coaches assisted the data collection activities but would not be allowed to provide any instruction, motivation, or feedback during the game. There was a 12-minute pause after each phase of the tournament, during which the participants could play free rondos.

Figure 1a shows the structure of the goalkeeper +3 vs. $3+$ goalkeeper configuration with goals of $6 \times 2 \mathrm{~m}$ (with goalkeepers). Figure $1 \mathrm{~b}$ presents the game 3 vs. 3 without goalkeepers, also played with $6 \times 2 \mathrm{~m}$ goals.
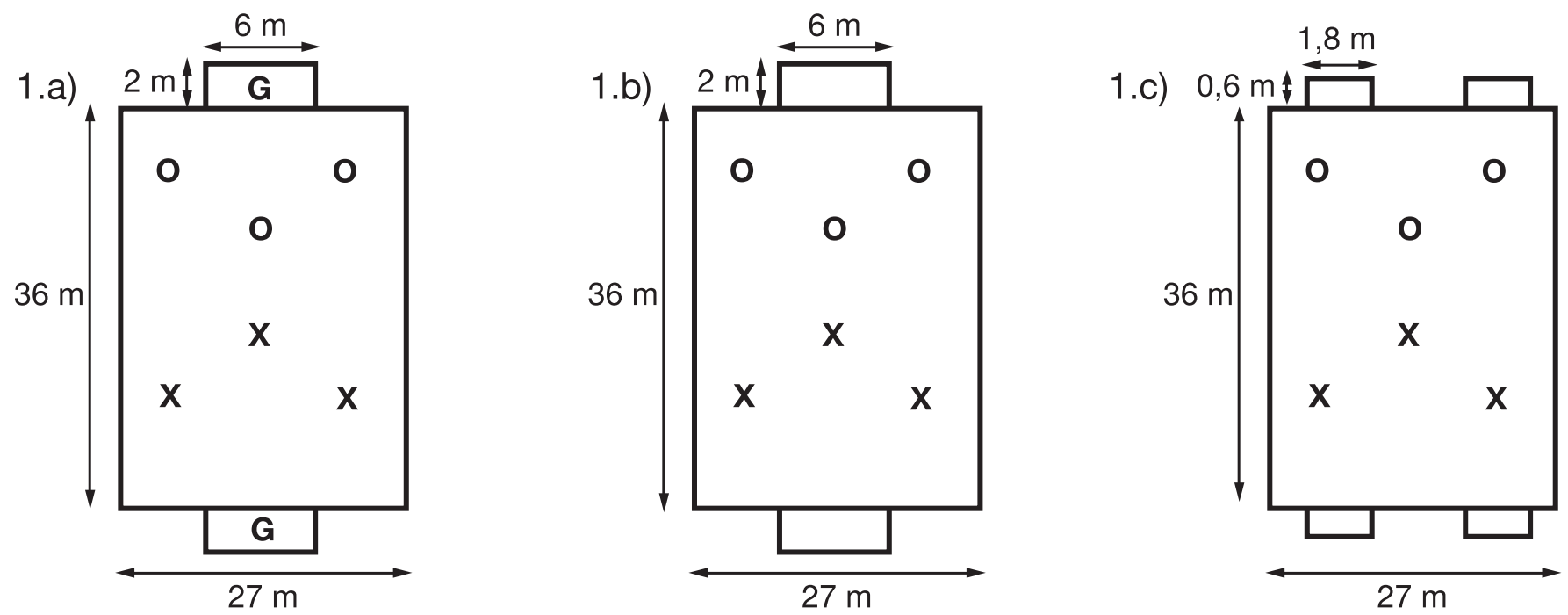

Figure 1. The analysed small-sided and conditioned game configurations 
Figure 1c depicts the game with 2 mini goals, which maintained the 3 vs. 3 structure but featured 2 mini goals of $1.80 \times 0.60 \mathrm{~m}$ on each goal line, positioned close to the sidelines. These games were also played without goalkeepers.

\section{Statistical analysis}

The results were presented as median and interquartile range (Q1-Q3). The Kolmogorov-Smirnov test was used to verify the distribution of data, with Friedman ANOVA applied for data with non-normal distribution. The significance level of $\alpha=0.05$ was adopted for all tests. The SPSS version 21 software (SPSS Inc., Chicago, USA) was used to perform all statistical tests. The effect size of Pearson $(r)$ served to verify the magnitude of the effect, for which the following classification was defined: small $(<0.29)$, medium (0.30-0.49), and large $(\geq 0.50)$, with respect to the calculation made with the following formula [21]:

$$
r=\frac{Z}{\sqrt{n}}
$$

In this formula, $Z$ is the $z$-score calculated by the SPSS software and $n$ is the size of the study values (that is, the total observation accomplished) on which $Z$ was based.

\section{Ethical approval}

The research related to human use has complied with all the relevant national regulations and institutional policies, has followed the tenets of the Resolution of the National Health Council (466/2012) and the Declaration of Helsinki, and has been approved by the Research Ethics Committee of Pedro Ernesto University Hospital at the Rio de Janeiro State University (HUPE/UERJ) and registered under the number 52519815.4.0000.5259.

\section{Informed consent}

Informed consent has been obtained from the legal guardians of all individuals included in this study. All participants have provided their assent forms.

\section{Results}

Table 1 shows that the configuration with goalkeepers provoked more offensive actions (total quantity) (medium effect) when compared with the game with 2 mini goals. Also, the number of movements in the back of the last opposing defender (depth mobility) (large effect) in offensive phases decreased compared with the game without goalkeepers. Playing without goalkeepers also led to a smaller number of movements that allowed to keep defensive stability in regions of ball dispute (balance) (medium effect). The same configuration finally increased movements that allowed the

Table 1. Median and interquartile range (Q1-Q3) of the quantity of offensive and defensive tactical principles performed in each configuration

\begin{tabular}{|c|c|c|c|c|c|c|c|}
\hline Tactical principles & With goalkeepers & Without goalkeepers & Two mini goals & $p$ & $\begin{array}{c}r \\
\mathrm{G} \times \mathrm{WG}\end{array}$ & $\begin{array}{c}r \\
\mathrm{G} \times \mathrm{MG}\end{array}$ & $\begin{array}{c}r \\
\mathrm{WG} \times \mathrm{MG}\end{array}$ \\
\hline \multicolumn{8}{|l|}{ Offensive } \\
\hline Penetration & $3.00(2.00-4.00)$ & $3.00(2.25-5.00)$ & $3.00(2.00-4.00)$ & 0.224 & 0.211 & 0.026 & 0.312 \\
\hline Offensive coverage & $5.00(3.00-7.00)$ & $6.00(4.00-8.00)$ & $5.00(3.00-6.75)$ & 0.618 & 0.096 & 0.035 & 0.199 \\
\hline Width and length & $16.47(13.00-20.00)$ & $15.00(10.00-19.00)$ & $14.00(10.00-18.75)$ & 0.094 & 0.194 & 0.390 & 0.090 \\
\hline Depth mobility & $2.00(1.23-4.00)^{*}$ & $1.00(0.00-2.00)$ & $2.00(1.00-3.00)$ & $<0.001$ & 0.610 & 0.257 & 0.353 \\
\hline Offensive unity & $7.00(4.00-8.75)$ & $6.00(4.00-9.00)$ & $5.00(3.25-7.00)$ & 0.337 & 0.126 & 0.284 & 0.154 \\
\hline Total offensive & 35.00 (30.00-38.00)\# & $31.50(28.25-35.75)$ & $30.00(27.00-34.00)$ & 0.337 & 0.999 & 0.425 & 0.273 \\
\hline \multicolumn{8}{|l|}{ Defensive } \\
\hline Delay & $7.00(5.00-8.75)$ & $7.00(6.00-9.00)$ & $8.00(5.00-9.00)$ & 0.431 & 0.075 & 0.137 & 0.128 \\
\hline Defensive coverage & $0.91(0.00-1.00) \#$ & $1.00(0.00-2.00)$ & $1.00(0.00-2.00)$ & 0.019 & 0.120 & 0.361 & 0.241 \\
\hline Balance & $6.00(5.00-8.00)^{*}$ & $4.00(3.00-6.00)$ & $5.00(3.00-7.75)$ & 0.011 & 0.417 & 0.112 & 0.305 \\
\hline Concentration & 7.89 (5.00-10.00) & $8.00(6.00-10.00)$ & $8.00(5.25-10.00)$ & 0.390 & 0.073 & 0.102 & 0.033 \\
\hline Defensive unity & 16.0 (13.00-19.00)\# & $17.00(13.25-20.75) \#$ & $13.00(9.00-16.75)$ & $<0.001$ & 0.032 & 0.490 & 0.522 \\
\hline Total defensive & $37.00(34.00-40.00)$ & $37.00(36.00-41.50) \#$ & $35.00(31.25-41.00)$ & 0.006 & 0.200 & 0.273 & 0.474 \\
\hline
\end{tabular}

* significant difference for the game without goalkeepers $(p<0.05)$

\# significant difference for the game with 2 mini goals $(p<0.05)$

$\mathrm{G}$ - small-sided and conditioned games with goalkeepers

WG - small-sided and conditioned games without goalkeepers

MG - small-sided and conditioned games with 2 mini goals 


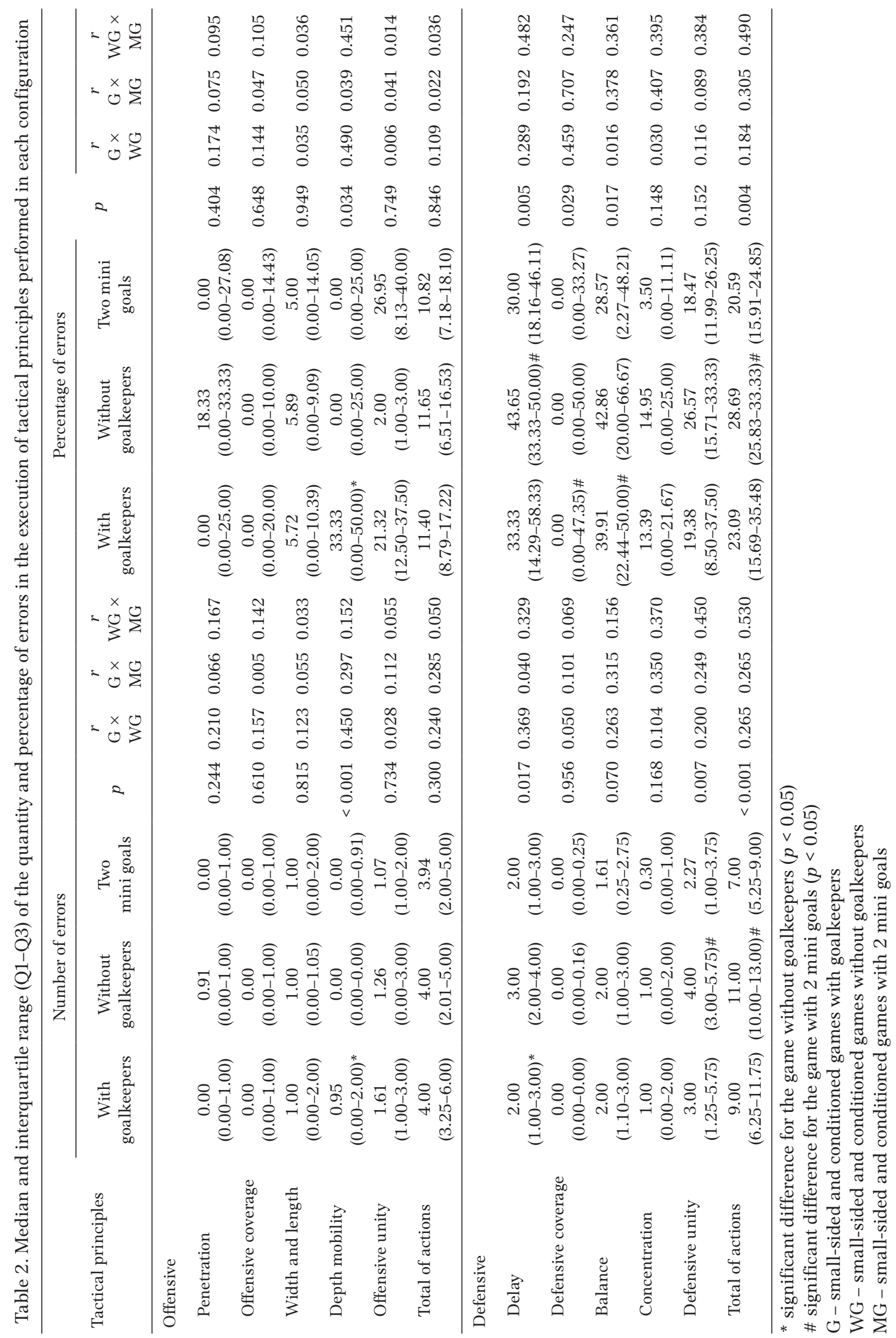


team to defend as a unit (defensive unity) (large effect) and the total of defensive actions (total quantity) (medium effect) compared with the game with 2 mini goals.

Table 2 shows the quantity and percentage of errors in the execution of tactical principles. It can be observed amongst the offensive principles that playing with goalkeepers made it more difficult to perform the movements in the back of the defence (depth mobility) (medium effect) when compared with the game without goalkeepers. As for the defensive principles, playing without goalkeepers made it more difficult to perform the movements in direct opposition to the ball carrier (delay) (medium effect), the movements that allowed to defend as a unit (defensive unity) (medium effect), and the defensive actions (large effect). The game with 2 mini goals decreased the percentage of errors of movements that supported the player who directly opposed the ball carrier (defensive coverage) (large effect) and movements that allowed to keep defensive stability in regions of ball dispute (balance) (medium effect).

It can be observed in Table 3 that playing without goalkeepers favoured strikes on goal in both offensive and defensive phases compared with other games (large effect). Playing with 2 mini goals led the team to earn fouls and win throw-ins and corners compared with the other games (medium effect). In the defensive phase, playing without goalkeepers provoked regaining ball possession more times when compared with the game with 2 mini goals (medium effect).

\section{Discussion}

This study analysed the influence of the absence of the goalkeeper and the number of goals in tactical actions in 3 configurations of SSCGs: (a) goalkeeper + 3 vs. 3 + goalkeeper; (b) 3 vs. 3 (no goalkeeper); and (c) 3 vs. 3 with 2 mini goals. The results show that manipulating task constraints causes trivial differences in behavioural patterns in SSCGs. The 3 configurations of SSCGs demonstrated more similarities than differences between them. A large and moderate effect size was observed for all comparisons that presented significant differences.

The movements in the back of the last defender of the opponent team in the games without goalkeepers decreased when compared with the game with goalkeepers. This probably happened because the attacking players performed more finishing actions (striking

Table 3. Median and interquartile range (Q1-Q3) of the result of actions performed in each configuration

\begin{tabular}{|c|c|c|c|c|c|c|c|}
\hline Result of actions & With goalkeepers & Without goalkeepers & Two mini goals & $p$ & $\begin{array}{c}r \\
\mathrm{G} \times \mathrm{WG}\end{array}$ & $\begin{array}{c}r \\
\mathrm{G} \times \mathrm{MG}\end{array}$ & $\begin{array}{c}r \\
\mathrm{WG} \times \mathrm{MG}\end{array}$ \\
\hline \multicolumn{8}{|l|}{ Offensive } \\
\hline Shoot at goal & $4.00(2.00-5.00)^{*}$ & 5.50 (5.00-6.00)\# & $3.00(2.00-4.00)$ & $<0.001$ & 0.505 & 0.429 & 0.934 \\
\hline $\begin{array}{l}\text { Keep possession } \\
\text { of the ball }\end{array}$ & $27.50(23.00-32.00)$ & 27.00 (24.00-32.00)\# & $25.50(23.00-30.00)$ & 0.045 & 0.151 & 0.227 & 0.378 \\
\hline $\begin{array}{l}\text { Earn a foul, win a corner } \\
\text { or throw-in }\end{array}$ & $1.00(0.00-3.00) \#$ & 2.00 (1.00-2.00)\# & $2.00(1.00-3.00)$ & 0.004 & 0.075 & 0.454 & 0.378 \\
\hline $\begin{array}{l}\text { Commit a foul, give away } \\
\text { a corner or throw-in }\end{array}$ & $2.00(1.00-3.00)$ & 1.00 (1.00-3.00)\# & $2.50(1.00-3.00)$ & 0.034 & 0.075 & 0.454 & 0.378 \\
\hline Loss of ball possession & $3.00(2.00-3.00)$ & 3.00 (2.00-4.00)\# & $2.50(1.00-3.00)$ & 0.050 & 0.050 & 0.227 & 0.328 \\
\hline \multicolumn{8}{|l|}{ Defensive } \\
\hline Regain ball possession & $3.00(2.00-3.00)$ & 3.00 (2.00-4.00)\# & $2.50(1.00-3.00)$ & 0.050 & 0.151 & 0.227 & 0.378 \\
\hline $\begin{array}{l}\text { Earn a foul, win a corner } \\
\text { or throw-in }\end{array}$ & $2.00(1.00-3.00)$ & 1.00 (1.00-3.00)\# & $2.50(1.00-3.00)$ & 0.034 & 0.075 & 0.454 & 0.378 \\
\hline $\begin{array}{l}\text { Commit a foul, give away } \\
\text { a corner or throw-in }\end{array}$ & 1.00 (0.00-3.00)\# & 2.00 (1.00-2.00)\# & $2.00(1.00-3.00)$ & 0.004 & 0.075 & 0.454 & 0.378 \\
\hline $\begin{array}{l}\text { Ball possession } \\
\text { of the opponent }\end{array}$ & $27.50(23.00-32.00)$ & 27.00 (24.00-32.00)\# & $25.50(23.00-30.00)$ & 0.045 & 0.151 & 0.227 & 0.378 \\
\hline Take a shot at own goal & $4.00(2.00-5.00)^{*}$ & $5.50(5.00-6.00) \#$ & $3.00(2.00-4.00)$ & $<0.001$ & 0.505 & 0.429 & 0.934 \\
\hline
\end{tabular}

* significant difference for the game without goalkeepers $(p<0.05)$

\# significant difference for the game with 2 mini goals $(p<0.05)$

$\mathrm{G}$ - small-sided and conditioned games with goalkeepers

WG - small-sided and conditioned games without goalkeepers

MG - small-sided and conditioned games with 2 mini goals 
L. Ometto I. Teoldo, D. Silva, F. Vasconcellos, Goals post change and tactical behaviour

on goal) from a medium to long distance to the goal, without having to dribble through the defensive live, since there was no goalkeeper to defend the goal. The studies conducted by Travassos et al. [2] and Clemente et al. [20] presented similar results in terms of the behavioural patterns because of the ease to reach the goals, which also shows that modifying goals directly interferes with the behavioural patterns adopted by the teams to attack or protect the objective of the game.

Another possible explanation for this to occur can be the positioning of the last opposing defender to be closer to the goal, which makes it difficult for attacking players to create movements that break the last defensive line. The literature indicates that in numerical inferiority, players move backwards to protect their own goal and compensate the effect of numerical inferiority $[22,23]$. Thus, players positioned closer to their own goal would seek to protect it and defend long distance strikes from the attacking team since there is no goalkeeper. Meanwhile, the 2 other advanced players divide the rest of the space of play between themselves and defend against the 3 attacking players. Thus, the absence of goalkeepers had an effect on the organization of the players on the field.

In addition, the play without goalkeepers decreased the number of movements that helped maintaining the defensive stability in the regions of ball dispute and increased movements that allowed the team to defend as a unit, as well as the total of defensive actions compared with the other games. As mentioned previously, the player who moves backward to perform the goalkeeper's job is then in a position that favours a higher frequency of movements that allow the team to defend as a unit [7]. However, this does not make these movements efficient. Players showed difficulty in decreasing the opponent's offensive amplitude and the space of play because of the absence of the rule of offside and of the goalkeepers [7].

On the other hand, a highlight of our results indicated that players increased the total of defensive actions. Playing with standard goals without goalkeepers increased possibilities of play for strikers compared with the game with 2 mini goals, which also raised the difficulty for the defenders to protect their goal and made them perform more defensive actions to compensate for the difficulty of the task [23, 24]. Also because of this, the players showed difficulty in performing these actions as they made more mistakes and were less efficient. This also implies that the games without goalkeepers made it difficult for the players to perform defensively. However, coaches can use such situations to create difficult conditions for the defenders who already perform well corresponding movements and synchronize with the goalkeeper.

Moreover, the defensive movements emphasize a behavioural pattern. For instance, the players who played forward (close to the ball carrier) probably positioned between the ball carrier and the goal to defend strikes on goal and consequently left the zones on the side of the field free. This behaviour is expected as part of the necessary adaptation to solve tasks in the best possible way [8]. However, the players also experienced difficulty in making the right movements of opposition against the ball carrier and were less efficient at performing them (quantity and quality of the actions), probably owing to the numerical inferiority, which possibly occurred in specific configurations without goalkeepers.

In contrast, the configuration featuring goalkeepers allowed an increase of the number of offensive tactical actions. This probably happened because of the presence of more players in the game. Thus, the players had more options of passes and more opportunities to move the ball between teammates with the objective to create opportunities to score goals. These results corroborate the findings of Silva et al. [24], who observed that SSCGs with more players led to a response where opponents were closer to the carrier of the ball (centre of play). This consequently generates more actions intended to pass the ball between teammates and also increases the number of offensive tactical actions in the game. Else, playing with goalkeepers does not allow strikes on goal from a medium or long distance and consequently leads players to passing the ball between teammates. Thus, players tend to pass the ball around with the intention to create goal opportunities.

In addition, playing with goalkeepers made the athletes perform more actions in the back of the last opposing defender. However, the players also made more mistakes in performing such movements. This higher rate of errors can be due to the higher number of actions in these games as there are more probabilities to make errors if one tries more times. Also, the players committed more fouls and gave the ball away through throw-ins and corner kicks in offensive phases compared with the games without goalkeepers, probably owing to the smaller area to protect in the presence of the goalkeeper. They would then be more assertive when defending. Following the same logic, in the defensive phase, the players suffered more fouls and earned more throw-ins and corner kicks in comparison with games without goalkeepers [24].

Lastly, playing with 2 mini goals generated tactical actions inside the centre of play. This could have hap- 
pened because of the positioning of the mini goals towards the wings and, consequently, a higher concentration of actions in the corridors. In these conditions, the exploration of effective space decreased, which favoured a higher proximity between players. In turn, more contact between the players provoked more fouls, throw-ins, and corners [25].

It is important to emphasize that a high variability of tactical actions occurs owing to the unpredictable nature of the game of soccer and because players of this age level tend to perform a higher number of actions associated with their respective position [26]. Consequently, players perform a higher number of specific tactical behaviours as they exhibit fewer other behaviours, which leads to a greater variability.

This study is important to demonstrate that, in practical terms, different configurations of games generate different tactical behaviours. These behaviours can be fostered with the aim to make it easier or more difficult to play in accordance with the objectives of the training session. Despite the advances presented in the understanding of the effect of modifications in goals on the tactical behaviour of soccer players, some limitations were observed. Amongst them, the participants were affiliated to the same team, which may have impacted on the way they played together or against one another. Moreover, the number of players (3 vs. 3 ) could also have influenced the tactical behaviour. Finally, the offside rule was not applied in SSCGs, as in similar studies. In accordance with the initial test protocol, the offside is not applied even if this results in contrasting with the internal logic of a formal soccer game.

New studies can continue verifying the effects caused in the tactical behaviour of players by modifications made to the goals, although without changes in the area covered by each player. Further research can also aim to investigate how long the players will take to comprehend determined tasks and acquire a continuous behavioural pattern, since studies related to periodization in soccer are lacking [27]. Finally, other studies can also verify if this pattern is maintained or if it alters depending on the age and individual characteristics of players [28]. Such information would contribute to the understanding of the effect of goal modifications on the tactical performance and behaviour of soccer players.

\section{Conclusions}

It is concluded that changes in the use of goals can influence the tactical actions of players in each SSCG configuration, especially that without goalkeepers, which provided more difficulty in managing the space of play and may cause more errors in performing core defensive tactical principles. On the other hand, the game with goalkeepers could have increased the total of offensive actions. Finally, the game with 2 mini goals led to play fragmentation as the athletes would position in the side corridors.

On the basis of these results, it is possible to hypothesize that there is an influence of goalkeepers in the play/training, both in defensive and offensive phases. In this context, it is then important to highlight that new methods of investigation must involve goalkeepers' presence in the assessment of the individual and collective responses since they are able to change the behaviour of the field players during training.

\section{Funding}

This study was funded by the State Department of Sport and Youth of Minas Gerais (SEESP-MG) in accordance with the State Act of Incentive to Sports, by FAPEMIG, CNPQ, FUNARBE, the Dean's Office for Graduate and Research Studies and the Centre of Life and Health Sciences at Universidade Federal de Viçosa, Brazil. The study was financed in part by Coordenação de Aperfeiçoamento de Pessoal de Nível Superior (CAPES), Brazil, finance code 001.

\section{Disclosure statement}

No author has any financial interest or received any financial benefit from this research.

\section{Conflict of interest}

The authors state no conflict of interest.

\section{References}

1. Tan TYH, Chow JY, Duarte R, Davids K. Manipulating task constraints shapes emergence of herding tendencies in team games performance. Int J Sports Sci Coach. 2017;12(5):595-602; doi: 10.1177/174795411 7727661.

2. Travassos B, Gonçalves B, Marcelino R, Monteiro R, Sampaio J. How perceiving additional targets modifies teams' tactical behavior during football small-sided games. Hum Mov Sci. 2014;38(1):241-250; doi: 10.1016/ j.humov.2014.10.005.

3. Sarmento H, Clemente FM, Harper LD, da Costa IT, Owen A, Figueiredo AJ. Small sided games in soccer - a systematic review. Int J Perform Anal Sport. 2018; 18(5):693-749; doi:10.1080/24748668.2018.1517288.

4. Clemente FM, Sarmento H. The effects of small-sided soccer games on technical actions and skills: a systematic review. Hum Mov. 2020;21(3):100-119; doi: 10.5114/hm.2020.93014. 
L. Ometto I. Teoldo, D. Silva, F. Vasconcellos, Goals post change and tactical behaviour

5. Araújo D. Tactical ability development: the role of constraints in decisional behavior [in Portuguese]. Motriz. 2009;15(3):537-540; doi: 10.5016/2942.

6. Clemente FM, Afonso J, Castillo D, Los Arcos A, Silva AF, Sarmento H. The effects of small-sided soccer games on tactical behavior and collective dynamics: a systematic review. Chaos Soliton Fract. 2020;134(1):109710; doi: 10.1016/j.chaos.2020.109710.

7. Teoldo I, Guilherme J, Garganta J. Training football for smart playing: on tactical performance of teams and players. Curitiba: Appris; 2017.

8. Davids K, Araújo D, Correia V, Vilar L. How small-sided and conditioned games enhance acquisition of movement and decision-making skills. Exerc Sport Sci Rev. 2013;41(3):154-161; doi: 10.1097/JES.0b013e318292 f3ec.

9. Silva P, Vilar L, Davids K, Araújo D, Garganta J. Sports teams as complex adaptive systems: manipulating player numbers shapes behaviours during football small-sided games. Springerplus. 2016;5(1):191; doi: 10.1186/ s40064-016-1813-5.

10. Silva DC, Costa VT, Casanova F, Clemente FM, Teoldo I. Comparison between teams of different ranks in small-sided and conditioned games tournaments. Int J Perform Anal Sport. 2019;19(4):608-623; doi: 10.1080/ 24748668.2019.1643598.

11. Bujalance-Moreno P, Latorre-Román PÁ, García-Pinillos F. A systematic review on small-sided games in football players: acute and chronic adaptations. J Sports Sci.2019;37(8):921-949;doi:10.1080/02640414.2018. 1535821.

12. Praça G, Barbosa GF, Murta C, Bredt SGT, Barreira D, Chagas $\mathrm{MH}$, et al. Influence of floaters and positional status on players' tactical, physical, and physiological responses in soccer small-sided games. Hum Mov. 2020; 21(3):54-63; doi: 10.5114/hm.2020.91346.

13. Machado JC, Barreira D, Teoldo I, Travassos B, Bosco Júnior J, Santos JOL, et al. How does the adjustment of training task difficulty level influence tactical behavior in soccer? Res Q Exerc Sport. 2019;90(3):403-416; doi: 10.1080/02701367.2019.1612511.

14. Teoldo I, Garganta J, Greco P, Mesquita I, Silva B, Müller E, et al. Analysis of tactical behaviours in smallsided soccer games: comparative study between goalposts of society soccer and futsal. Open Sports Sci J. 2010;3(1):10-12; doi: 10.2174/1875399X01003010010.

15. Castellano J, Silva P, Usabiaga O, Barreira D. The influence of scoring targets and outer-floaters on attacking and defending team dispersion, shape and creation of space during small-sided soccer games. J Hum Kinet. 2016;51(1):153-163; doi: 10.1515/hukin-2015-0178.

16. Ometto L, Alkmim MNR, Praca GM, Vasconcellos F. What are the most important field zones when goalposts are manipulated in soccer? Hum Mov. 2018;19(5, special issue):106-113; doi: 10.5114/hm.2018.81015.

17. Teoldo I, Garganta J, Greco PJ, Mesquita I, Maia J. System of tactical assessment in soccer (FUT-SAT): devel- opment and preliminary validation. Motricidade. 2011; 7(1):69-83; doi: 10.6063/motricidade.121.

18. Köklü Y, Sert Ö, Alemdaroğlu U, Arslan Y. Comparison of the physiological responses and time-motion characteristics of young soccer players in small-sided games: the effect of goalkeeper. J Strength Cond Res. 2015;29(4): 964-971; doi: 10.1519/JSC.0b013e3182a744a1.

19. Mallo J, Navarro E. Physical load imposed on soccer players during small-sided training games. J Sports Med Phys Fitness. 2008;48(2):166-171.

20. Clemente FM, Wong DP, Martins FML, Mendes RS. Acute effects of the number of players and scoring method on physiological, physical, and technical performance in small-sided soccer games. Res Sports Med. 2014;22(4):380-397; doi: 10.1080/15438627.2014. 951761.

21. Cohen J. A power primer. Psychol Bull. 1992;112(1): 155-159; doi: 10.1037//0033-2909.112.1.155.

22. Ric A, Hristovski R, Gonçalves B, Torres L, Sampaio J, Torrents C. Timescales for exploratory tactical behaviour in football small-sided games. J Sports Sci. 2016; 34(18):1723-1730; doi: 10.1080/02640414.2015.113 6068.

23. Vilar L, Araújo D, Davids K, Travassos B, Duarte R, Parreira J. Interpersonal coordination tendencies supporting the creation/prevention of goal scoring opportunities in futsal. Eur J Sport Sci. 2014;14(1):28-35; doi: 10.1080/17461391.2012.725103.

24. Silva B, Garganta J, Santos R, Teoldo I. Comparing tactical behaviour of soccer players in 3 vs. 3 and 6 vs. 6 small-sided games. J Hum Kinet. 2014;41(1):191-202; doi: 10.2478/hukin-2014-0047.

25. Silva P, Esteves P, Correia V, Davids K, Araújo D, Garganta J. Effects of manipulations of player numbers vs. field dimensions on inter-individual coordination during small-sided games in youth football. Int J Perform Anal Sport. 2015;15(2):641-659; doi: 10.1080/ 24748668.2015.11868821.

26. Silva RNB, Teoldo I, Silva DC, Ferreira LA, Dos-Santos JW. Influence of the positional role on the tactical actions of soccer players [in Portuguese]. Pensar Prát. 2018;21(3):672-682; doi: 10.5216/rpp.v21i3.50392.

27. Afonso J, Bessa C, Nikolaidis PT, Teoldo I, Clemente FM. A systematic review of research on tactical periodization: absence of empirical data, burden of proof, and benefit of doubt. Hum Mov. 2020;21(4):37-43; doi: 10.5114/hm.2020.95329.

28. Sarmento H, Marques A, Field A, Martins J, Gouveia ÉR, Mondagrón LP, et al. Genetic influence on football performance: a systematic review. Hum Mov. 2020;21(4): 1-17; doi: 10.5114/hm.2020.94198. 